\title{
Efficacy of Intralesional Purified Protein Derivative for Cutaneous Warts: A Meta-analysis
}

\author{
Sarah Faye V. Obbus, ${ }^{1}$ Jay-V James G. Barit ${ }^{1}$ and Claudine Yap-Silva ${ }^{2}$ \\ ${ }^{1}$ Section of Dermatology, Department of Medicine, Philippine General Hospital, University of the Philippines Manila \\ ${ }^{2}$ Section of Dermatology, Department of Medicine, College of Medicine and Philippine General Hospital, University of the Philippines Manila
}

\begin{abstract}
Introduction. Intralesional purified protein derivative (PPD) is an affordable therapeutic option that has been studied for cutaneous warts. However, the lack of good evidence precludes its widespread use.

Objective. To determine the efficacy and safety of intralesional PPD in the treatment of cutaneous warts.

Methods. A systematic search for controlled clinical trials comparing intralesional PPD and placebo or any conventional therapy was conducted using electronic databases. The included studies were assessed for risk of bias, and data such as clearance rate of target and distant lesions, recurrence rate, and adverse events were extracted. Analysis was done through RevMan v5.3.

Results. Four controlled clinical trials composed of 205 patients were included. All of the studies compared intralesional PPD to placebo as comparator. Intralesional PPD had a significantly higher clearance rate of target wart $(R R=0.43[0.22,0.84], P=0.01)$ and a significantly higher clearance rate of distant lesions $(R R=0.59[0.41,0.85]$, $\mathrm{P}=0.005)$ as compared to placebo. However, there was no significant difference in the recurrence rate $(R R=0$ $[-0.07,0.07], \mathrm{P}=0.98)$. Adverse events reported were only considered minor.
\end{abstract}

Conclusion. Intralesional PPD is an effective and safe treatment option for cutaneous warts. However, more well-structured RCTs with longer follow-up period and those comparing it with conventional treatment are needed to further support its use.

Key Words: purified protein derivative, warts, meta-analysis, PPD, verruca

\section{INTRODUCTION}

E-poster presented at the $40^{\text {th }}$ Annual Convention of the Philippine Dermatological Society, November 8-10, 2017, EDSA Shangri-La, Mandaluyong City, Philippines.

Corresponding author: Sarah Faye V. Obbus, MD

Section of Dermatology

Department of Medicine

Philippine General Hospital

Taft Avenue, Manila 1000, Philippines

Telephone: +6325548400 local 5105/5106

Email: sfobbus@yahoo.com

\section{Cutaneous warts and management}

Cutaneous warts are a common reason for consult among dermatologists. It is one of the top 10 diagnoses made at the Philippine General Hospital Section of Dermatology OPD for the past three years (2014-2016). These are benign epithelial proliferations occurring in both skin and mucous membranes that are caused by different strains of human papillomaviruses. ${ }^{1}$

These warts can spontaneously resolve after a few months or years in children and even in immunocompetent adults, albeit with a very high recurrence rate. ${ }^{2}$ Many options are available and are being used in the treatment for this condition, which highlights the fact that there is really not one among these that is directly an antiviral treatment and is consistently and uniformly effective. ${ }^{1}$ Examples of commonly used methods are chemical destruction, physical destruction with electrodessication or cryotherapy and immunotherapy. ${ }^{1}$ These treatment options vary in effectiveness, ease of application, cost and side effects. 
Intralesional PPD for Cutaneous Warts

\section{Purified protein derivative (PPD) and how it works}

Many antigens with variable treatment outcomes have been used and studied as a form of intralesional therapy, including Candida albicans, measles, mumps and rubella (MMR), Bacillus Calmette-Guerin, and PPD, among others. ${ }^{3}$ How these antigens work has not been fully elucidated; however, there are a number of theories that try to explain the phenomenon. One such theory is that the antigen stimulates a delayed-type hypersensitivity or a systemic $T$-cell mediated response which promotes the release of cytokines including interferon-gamma and interleukin 2, thereby increasing the ability of the immune system to clear the human papillomaviruses. Another theory argues that even the trauma induced by the injection of antigens such as PPD is enough to stimulate a response from the immune system..$^{2,4,5}$

\section{Significance of the Study}

Cutaneous warts are a common condition across different populations; thus, there is a need for an intervention that is effective, convenient, safe and affordable. Although there are a lot of therapeutic options for warts, these options can be painful and inconvenient to use due to frequency of application and can lead to scarring, and can also have a high recurrence rate. ${ }^{4}$ Most of these therapeutic options also only have an effect locally; ${ }^{1}$ thus, treatment should be applied on each wart making it inconvenient especially when a person presents with multiple and disseminated warts. The use of intralesional PPD can be a good therapeutic option if proven to be effective and safe due to its systemic effect and its low cost. Despite these potential benefits, it is the unavailability of good quality studies proving its effectiveness that preclude its worldwide use.

To date, there are no published systematic reviews and meta-analysis on the use of intralesional purified protein derivative in the treatment of warts.

\section{OBJECTIVES}

\section{General Objective}

- To assess the efficacy and safety of intralesional purified protein derivative in the treatment of cutaneous warts in comparison to placebo and other therapeutic options.

\section{Specific Objectives}

- To determine the complete clearance rate of the target wart when using intralesional PPD as compared to placebo and other interventions

- To determine the complete clearance rate of distant warts when using intralesional PPD as compared to placebo and other interventions

- To determine the side effects related to the use of intralesional PPD in the treatment of warts

- To determine the recurrence rate of cutaneous warts after treatment with intralesional PPD

\section{METHODS}

\section{Inclusion and exclusion criteria}

As for the types of study included, only controlled clinical trials comparing intralesional PPD (regardless of the dosing used, frequency of administration, and whether injected to the mother wart only or all warts) with other conventional therapies and placebo in the treatment (complete clearance rate) of cutaneous warts were reviewed. No exclusion criteria were made on the type of cutaneous warts that the population have, recalcitrance to treatment, duration of disease, number of treatment options previously tried, and number of lesions.

\section{Search method}

The search for eligible studies was done using different electronic databases. These include PubMed/Medline, The Cochrane Library, HERDIN, Google Scholar and the electronic database of the Journal of American Academy of Dermatology. The keywords ["warts" OR "verruca"] and ["tuberculin" OR "PPD” OR "purified protein derivative"] connected by the operator AND were used in the search.

\section{Data collection and analysis}

\section{Selection of studies}

All search results from the different electronic databases were first screened by scanning the titles and abstracts to check whether these studies fulfilled the inclusion criteria. For unclear articles, full-text were retrieved and reviewed. Duplicate studies were also identified and marked. After the initial screening, the full-text of all the included studies were retrieved to confirm whether these studies were eligible or not. These steps were done by two independent authors, and disagreements were resolved by discussion.

\section{Data extraction}

For each included study, the two independent authors extracted the needed set of data. The specific data gathered were extracted using the software RevMan 5.3. ${ }^{6}$ Data retrieved included the title of the study, design of the trial, inclusion and exclusion criteria, characteristics of the population, the intervention including the dose, dosing frequency/schedule and duration, the comparator, assessed outcomes and the corresponding figures, number of dropouts, adverse events recorded, recurrence rates, follow-up schedule, and country of origin of the study.

\section{Assessment of Risk of Bias}

The internal validity for each individual study was assessed by two independent authors guided by Cochrane Collaboration's tool for assessing of risk bias (Table 8.5.a) of the Cochrane Handbook for Systematic Reviews of Interventions. ${ }^{7}$ Each study's validity was assessed by using different domains such as randomization, allocation 
concealment, blinding of participants and personnel, blinding of outcome assessment, incomplete data, selective reporting and other bias with the criteria of judgment outlined in Table 8.5.d from the same handbook. ${ }^{7}$ For each domain, each study was categorized to be either high-risk, low-risk, or with unclear risk. Any differences in the bias assessment between the two authors were resolved and settled by discussion between the two.

\section{Measuring Treatment Effect}

Since the outcome measured in the studies were dichotomous - complete vs incomplete clearance of target and distant lesions, and the recurrence or non-recurrence of warts, risk ratio was computed for each. Meta-analysis was then done on these data using the software Review Manager 5.3. ${ }^{6} \mathrm{~A}$ p-value of less than 0.05 was considered significant.

\section{Missing or unavailable data}

Since not all studies had the outcome measures computed, only those with available data for analysis were included in the secondary outcomes.

\section{Data synthesis}

Descriptive analysis was done first by comparing the population, intervention, comparator, outcomes and methodology or the design of the studies included. Data was then statistically analyzed using the Review Manager 5.3 software. $^{6}$ Risk ratios, p-values, confidence intervals, and heterogeneity data were extracted from this analysis. A subgroup analysis on the outcomes of the use of intralesional PPD vs placebo alone was done, while data on other comparators were discussed separately.

Heterogeneity was assessed by looking at the forest plot and getting the value of the $\mathrm{I}^{2}$ statistic. Cochrane Handbook for Systemic Interventions Section 9.5 was used as a guide to interpret this data. ${ }^{7}$

\section{RESULTS}

\section{Description of Studies}

\section{Search Results}

A total of 35 articles were obtained through electronic searches: PubMed (11), Cochrane (6), and Google Scholar (18). No trials were identified in both HERDIN and JAAD. There were a total of 8 duplicates identified, and 21 irrelevant or ineligible studies were exluded by reading the titles and abstracts. The full copy of the remaining 6 studies were then retrieved and assessed. Two of the studies were excluded since they were not controlled clinical trials. A total of four studies met the inclusion criteria set by this meta-analysis.

\section{Included Studies}

Details on the four studies which fulfilled the inclusion criteria are summarized in Table 1.

\section{Setting}

All studies were single-center studies and patients were recruited from the outpatient department of different hospitals. Abd-Elazeim's study and Sharquie's study were done in Egypt and Iraq, respectively. The other two did not specifically mention the country of origin, but the authors had common affiliations - Ain Shams University in Cairo, Egypt for Shaheen's study while in Amirnia's study, the authors were from different institutions in Tabriz, Iran.

\section{Population}

The total number of participants in all the studies was 202, with majority coming from the Amirnia's study. Sample sizes ranged from 30 (Shaheen 2015 and Sharquie 2016) to 102 (Amirnia 2015). The population of three of the studies had a mean age of greater than 20. It was only the Sharquie's study which had a relatively younger population with a mean age of 13.53. Male to female ratio was at 1.06:1 (104:98). Two of the studies (Shaheen 2015 and Sharquie 2016) classified their patients according to types of verruca vulgaris, with common warts as the most prevalent, while the other two studies (Abd-Elazeim 2014 and Amirnia 2015) described the locations of the lesions - with the limbs being the most common. Across all studies, there was no significant difference in the baseline characteristics in the intervention and control arms.

In both Abd-Elazeim's and Amirnia's study, only patients with recalcitrant warts were included. However, it was only in Amirnia's study where recalcitrant was defined: disease duration of at least two years and unresponsive to at least one conventional treatment modality. As for the inclusion criteria, only Sharquie's test did not specify the need for an existing immunity against the antigen. In the other three studies, each participant had to undergo an intradermal PPD testing, and a negative result conferred exclusion from the study. Other pertinent exclusion criteria included concomitant treatment for warts, iatrogenic or primary immunosuppression, anaphylaxis, pregnancy and lactation, allergic skin diseases, asthma, and generalized dermatitis.

\section{Treatment arm}

Although all of the studies used purified protein derivative given intralesionally, the dosage, frequency, and target wart differed from each study excluding AbdElazeim's and Amirnia's studies which patterned their dosing and frequency of application on the method described by Johnson, et.al ${ }^{8}$ in their study on the intralesional injection of mumps or Candida skin test antigens. They injected the amount of PPD depending on the result of the intradermal testing. If the diameter was less than $20 \mathrm{~mm}, 0.3 \mathrm{~mL}$ of intralesional PPD was injected, $0.2 \mathrm{~mL}$ if diameter was between $21 \mathrm{~mm}$ and $40 \mathrm{~mm}$, and $0.1 \mathrm{~mL}$ if greater than $40 \mathrm{~mm}$. Although Shaheen's study also depended on the intradermal test result for the amount of PPD injected, different values as cut-off for each amount was used. If 
Table 1. Summary of the four included studies

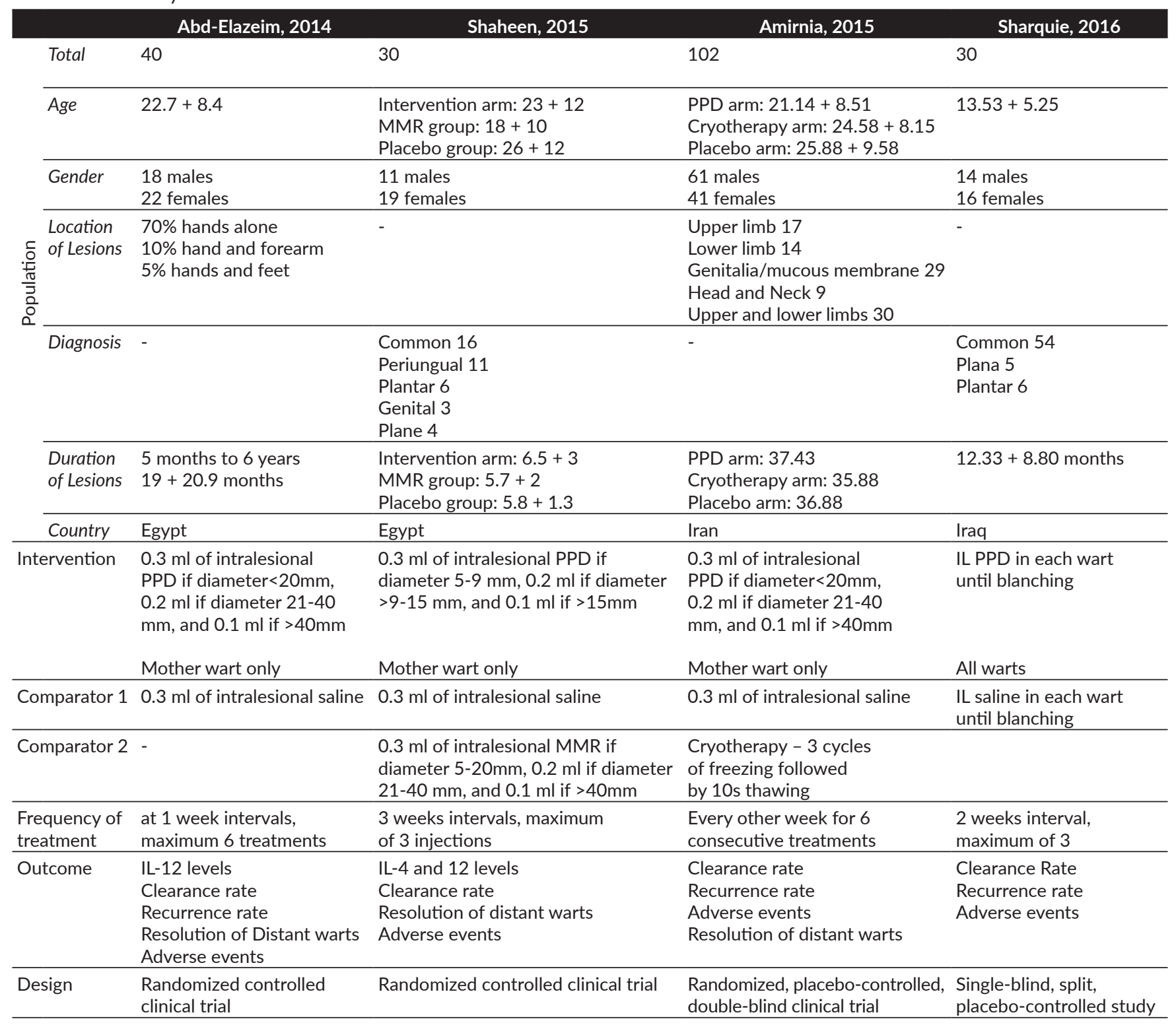

the diameter was $5-9 \mathrm{~mm}, 0.3 \mathrm{~mL}$ was injected, $0.2 \mathrm{~mL}$ if diameter was more than $9 \mathrm{~mm}$ to $15 \mathrm{~mm}$ and $0.1 \mathrm{~mL}$ if diameter was greater than $15 \mathrm{~mm}$. Only Sharquie's study did not specify the amount of intervention given; instead, they used the amount that was needed for each wart to blanch, varying the amount per wart. Also, it was only in Sharquie's study where every wart in one half of the body of the patient (split-study) was injected with the said solution. In the other three, only the mother wart or the largest wart was injected with PPD.

As for the frequency of dosing, Amirnia 2015 and Abd Elazeim 2014 injected their patients every other week for a maximum of 6 treatments. Shaheen 2015, on the other hand, injected at three week intervals and maximum of 3 treatments, while Sharquie 2016 had injections at 2 weeks intervals with a maximum of 3 treatments.

\section{Control Group}

\section{Placebo}

All studies used intralesional saline as placebo, injected at the same frequency as that of the treatment arm. The amount did not differ though depending on the result of the intradermal PPD as compared to the PPD group. Sharquie 2016 again used blanching as a measure of the amount that was injected on all warts on the particular half of the body (split study), while the other three gave a standard measurement of $0.3 \mathrm{~mL}$ on the mother wart.

\section{Other comparators}

Shaheen 2015 used another comparator which was intralesional measles, mumps, rubella (MMR) at a dose depending on the intradermal MMR test $-0.3 \mathrm{~mL}$ if the 
diameter was less than $20 \mathrm{~mm}, 0.2 \mathrm{~mL}$ if diameter was between 21 and 40 , and $0.1 \mathrm{~mL}$ if greater than $40 \mathrm{~mm}$. This was also given at the same frequency as that of placebo and intralesional PPD.

Amirnia 2015 also had another intervention being compared to intralesional PPD. A set of patients was assigned to the cryotherapy arm, receiving 3 cycles of freezing followed by 10 seconds thawing per session, done every other week for a total of 6 sessions. This was also only applied on the mother wart.

\section{Outcome detection}

Each study evaluated the warts injected every session and after the study if there was complete resolution/disappearance, good response (50-99\%), minimal/poor response ( $<50 \%)$, and no response/no change from baseline. The three studies which only injected the mother wart (Abd-Elazeim 2014, Amirnia 2015, and Shaheen 2015) also categorized the response of distant warts to either complete, minimal, poor, and no response. The number of patients who developed recurrence on the previous site or new sites after 6 months was also reported by Abd-Elazeim 2014 and Amirnia 2015 and after 2 months for Sharquie 2016.

Two of the studies included the measurement of interleukin levels after injection of intralesional PPD - IL12 for Abd-Elazeim 2014 while IL-12 and IL-4 levels for Shaheen 2015.

\section{Outcomes reported}

\section{Clearance rate of target wart}

All of the studies reported cure using dichotomous outcomes - clearance versus no clearance. They reported them as rates of complete, partial, or minimal/poor response of the target lesions. The complete clearance rates for intralesional PPD was significantly higher than placebo in all the studies. Shaheen 2015 furthermore found no significant difference with the clearance rate of intralesional MMR while Amirnia 2015 reported better clearance rates with intralesional PPD than cryotherapy.

\section{Clearance rate of distant wart}

Three of the studies (Abd-Elazeim 2014, Amirnia 2015, and Shaheen 2015) also reported the clearance rates of distant warts. Sharquie 2016, on the other hand, did not report this since they injected all lesions on one side of the body with intralesional PPD. Also, since it was a split-study, the systemic effect of intralesional PPD might overlap with the systemic and local effect of the placebo.

\section{Recurrence rate}

Rates of appearance of new lesions after 6 months on either the previous site or on new sites were reported by both Abd-Elazeim 2014 and Amirnia 2015. Sharquie 2016, on the other hand, had a follow-up of 2 months only.

\section{Adverse events}

Each study enumerated the adverse events reported by the patients in all treatment groups. It was only Sharquie 2016 though which added a qualifier to pain as either necessitating the intake of non-steroidal anti-inflammatory drugs or not.

\section{Design}

Three out of the four studies (Abd Elazeim 2014, Shaheen 2015, and Amirnia 2015) were randomized controlled clinical trials with intralesional saline as placebo. Shaheen 2015 had the addition of intralesional MMR as another comparator, while Amirnia 2015 had another group of patients not included in the randomization process to receive cryotherapy. Sharquie 2016, on the other hand, was a split, placebo-controlled study, with each participant enrolled in the trial receiving both intralesional PPD and intralesional saline.

\section{Excluded studies}

After the preliminary screening, many relevant studies were still excluded because most of them were uncontrolled studies. A total of 6 articles were retrieved in the end, however, only four were included in this study. The two excluded studies (Kerure AS 2016 and Kus 2004) were only letters to the editors which even though detailed their trials using intralesional PPD on warts, were uncontrolled clinical studies.

\section{Risk of Bias in Included Studies (Figure 1)}

\section{Selection Bias}

Three of the four studies (Abd-Elazeim 2014, Shaheen 2015, and Amirnia 2015) were assessed to have an unclear risk for the item on random sequence generation since even if the studies stated or claimed that randomization was done, there were no mention on the specific method that was used for each study that would support the claim. The same goes for allocation concealment since there was also no mention of any techniques done to assure this. These techniques are usually done to reduce the selection bias, making the baseline characteristics of the different treatment arms similar. Although Sharquie 2016 did not use these techniques, the authors still assessed this study to have low risk for selection bias since this was a split study the same person received both the intervention and control, making the baseline characteristics exactly the same.

\section{Performance Bias and Detection Bias}

Amirnia 2015 study was assessed to have low risk for performance and detection bias since it detailed how they tried to blind the patients and the examiner. Every patient underwent intradermal PPD testing and injections were made with similar looking syringes. Sharquie 2016, on the other hand, was assessed to have an unclear risk in performance 


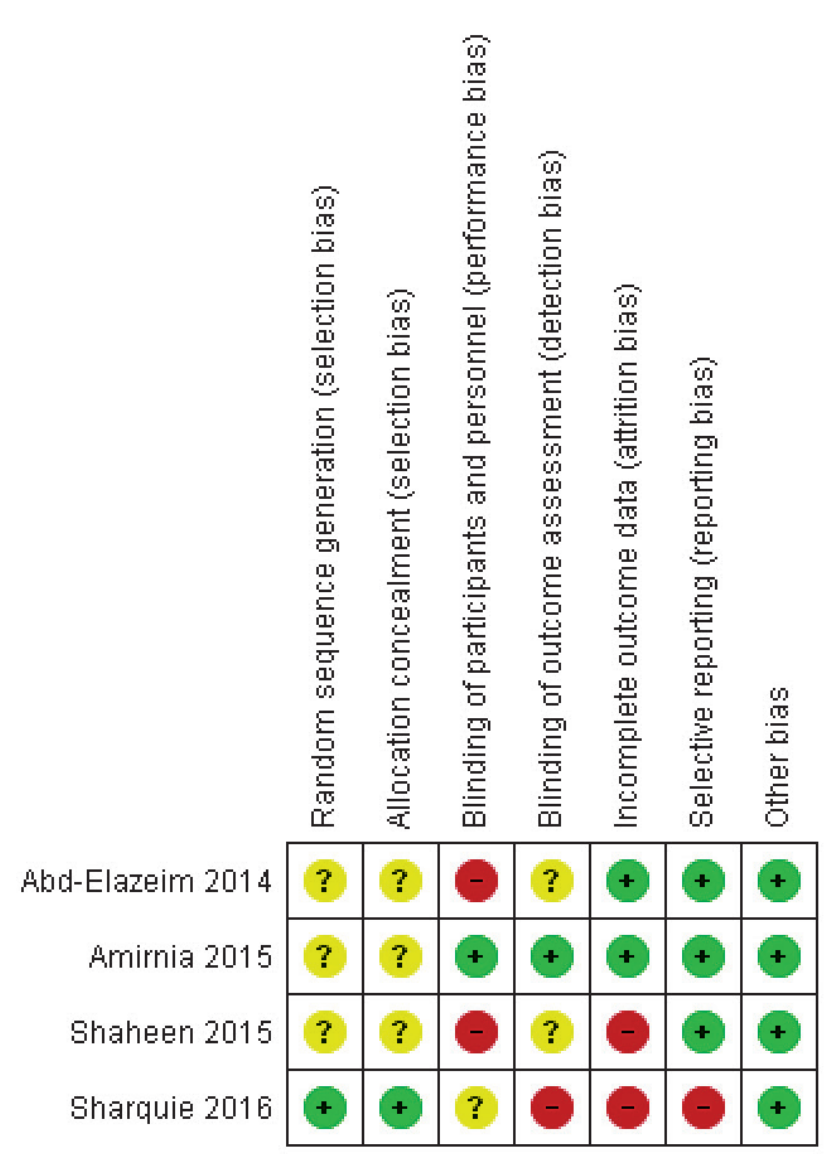

Figure 1. Risk of bias summary showing the authors' assessment on each domain and for each included study.

bias and with high risk in detection bias since they only did a single-blind study, but no details were disclosed on how this went about. Shaheen 2015 and Abd-Elazeim were both high risk in performance bias since only the treatment groups received the intradermal $\mathrm{PPD} / \mathrm{MMR}$ testing at the start. Hence, no blinding was done for the patients. As for the detection bias, there was no mention of another blinded assessor and thus was assessed to have unclear risk.

\section{Attrition bias}

There were no drop-outs in the Abd-Elazeim 2014 study; thus, it was assessed to have low risk for attrition bias. In Amirnia's study, there were no drop-outs in the intervention group, but the intralesional group had 1 dropout $(2.8 \%)$ while the cryotherapy group had 2 drop-outs (5.7\%). However, these percentages were not significant, making this study low-risk for attrition bias. Shaheen's study, however, mentioned that there were originally 38 participants in the study but only 30 finished the study. It did not mention which groups the 8 participants (21\%), who withdrew due to personal reasons, came from. They were also not included in the analysis, making this study at high risk for attrition bias. Sharquie 2016 also had 11 dropouts $(26.8 \%)$ which was a significant percentage and again they were not included in the analysis, thus, this study was assessed to be at high risk for attrition bias.

\section{Reporting Bias}

With the exception of Sharquie's study, all the other studies were assessed to be of low risk for this bias since all the needed information and outcomes that were part of their objectives from the start of the study were reported. Sharquie's study, on the other hand, omitted the reasons for the high number of drop-outs and just proceeded with the analysis using only the number of participants who finished the trial. These could have affected the number of adverse events reported for each intervention. It was then assessed to be at high risk for reporting bias.

\section{Other biases}

All studies were assessed to have low risk for other potential bias. No studies reported conflict of interests nor were funded by pharmaceutical companies. There were also no deviations found from the study protocol on all studies. There were no mention though on how the different authors tried to minimize the possibility of patients using another therapeutic option which could act as a contaminant, and on how they could have addressed this issue if the event arises. Nevertheless, the authors still committed that these studies were of low risk for other biases.

\section{Data and Pooled Analysis}

All studies were included in the meta-analysis, but only the data of the intervention (intralesional PPD) group and placebo group were included for Shaheen 2015 and Amirnia 2015. All of these studies compared intralesional PPD and intralesional saline/placebo. Effect estimates to the left of the vertical line of the forest plot implies benefit from the use of intralesional PPD vs placebo.

\section{Incomplete resolution of target wart (Figure 2)}

The risk ratio of having incomplete resolution of the target wart is at $0.43(\mathrm{p}=0.01)$ with both ends of the estimate $(0.22,0.84)$ at the left of the null value, implying benefit of intralesional PPD over placebo. The random effects model was used for this outcome due to the high heterogeneity of the studies $(87 \%)$.

\section{Incomplete clearance of distant warts (Figure 3)}

For the outcome of incomplete clearance of distant warts, only three studies were included because the methodology of Sharquie's study made this impossible to measure - split study, each patient receiving both intralesional PPD and saline. Risk ratio was at $0.59(\mathrm{p}=0.005)$, and again both ends of the $95 \%$ confidence interval $(0.41,0.85)$ were on the left side of the null value implying benefit of using intralesional PPD versus placebo. Heterogeneity was also high at $60 \%$. 


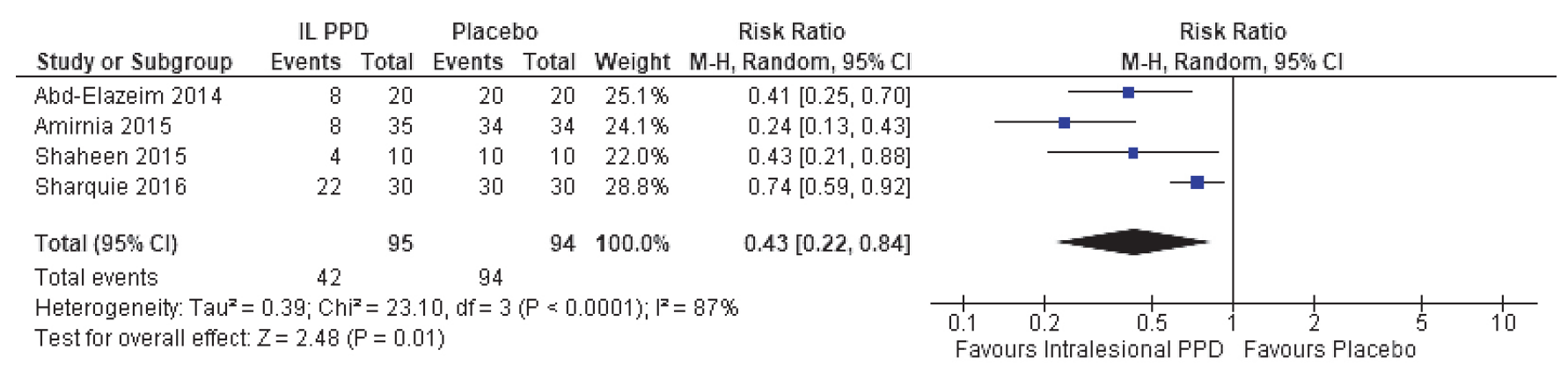

Figure 2. Forest plot of the outcome: incomplete resolution of target wart including 4 studies.

\begin{tabular}{|c|c|c|c|c|c|c|c|c|c|c|}
\hline \multirow[b]{2}{*}{ Study or Subgroup } & \multicolumn{2}{|c|}{ IL PPD } & \multicolumn{2}{|c|}{ Placebo } & \multirow[b]{2}{*}{ Weight } & \multirow{2}{*}{$\begin{array}{c}\text { Risk Ratio } \\
\text { M-H, Random, } 95 \% \mathrm{Cl}\end{array}$} & \multirow{2}{*}{\multicolumn{4}{|c|}{$\begin{array}{c}\text { Risk Ratio } \\
\text { M-H, Random, } 95 \% \mathrm{Cl}\end{array}$}} \\
\hline & Events & Total & Events & Total & & & & & & \\
\hline Abd-Elazeim 2014 & 15 & 20 & 20 & 20 & $44.5 \%$ & $0.76[0.58,0.98]$ & & $\rightarrow$ & & \\
\hline Amirnia 2015 & 17 & 35 & 32 & 34 & $37.7 \%$ & $0.52[0.36,0.73]$ & & & & \\
\hline Shaheen 2015 & 4 & 10 & 10 & 10 & $17.8 \%$ & $0.43[0.21,0.88]$ & & & & \\
\hline Total $(95 \% \mathrm{Cl})$ & & 65 & & 64 & $100.0 \%$ & $0.59[0.41,0.85]$ & & & & \\
\hline Total events & 36 & & 62 & & & & & & & \\
\hline $\begin{array}{l}\text { Heterogeneity: Tau } \\
\text { Test for overall effec }\end{array}$ & $\begin{array}{l}0.06 ; \text { Chi } \\
z=2.81\end{array}$ & $\begin{array}{l}P=5.02 \\
P=0.0\end{array}$ & $\begin{array}{l}2, d f=2( \\
005)\end{array}$ & $P=0.08$ & $8) ; I^{2}=609$ & & 0.01 & $\begin{array}{c}0.1 \\
\text { Favours IL PPD }\end{array}$ & $\begin{array}{c}10 \\
\text { Favours Placebo }\end{array}$ & 100 \\
\hline
\end{tabular}

Figure 3. Forest plot of the outcome: incomplete resolution of distant warts including 3 studies.

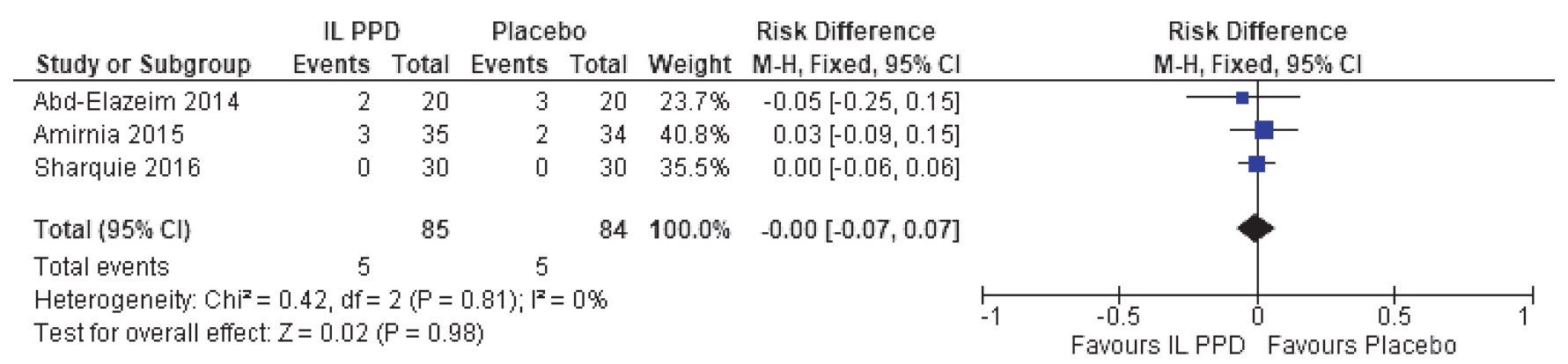

Figure 4. Forest plot of the outcome: recurrence rate including 3 studies.

\section{Recurrence rates (Figure 4)}

Only three out of the four studies included data on recurrence rates. Abd-Elazeim 2014 and Amirnia 2015 followed up their patients up to 6 months while for Sharquie 2016, 2 months.

Since Sharquie 2016 reported no events of recurrence for both groups, risk difference over risk ratio was used so that the study will be included in the estimation process. Fixed effects model was used because of the low heterogeneity value. The computed risk difference was at $0(\mathrm{p}=0.98)$, the null value, with the ends of the confidence interval $(-0.07$, 0.07 ) at both sides of the forest plot implying no significant difference between intralesional PPD and placebo when it comes to recurrence rates.

\section{Versus measles, mumps, rubella}

In Shaheen's study, there was no significant difference between intralesional PPD and MMR when it comes to incomplete resolution of target wart (RR: 2, p-value >0.05) and distant wart (RR: 0.67, p-value> 0.05).

\section{Versus cryotherapy}

In Amirnia's study, intralesional PPD had significantly lower rates of incomplete resolution of target wart (RR: 0.28 , $\mathrm{p}$-value $<0.001)$ and distant wart (RR 0.486, $\mathrm{p}$-value $<0.001)$ when compared to cryotherapy. However, there was no significant difference when it comes to the recurrence rates (RR: 0.355, p-value 0.08).

\section{Adverse events}

As for adverse events, the different studies enumerated these together with the incidence rate. However, AbdElazeim 2014 and Sharquie 2016 only mentioned the side effects complained by the patients in the intervention arm. Abd-Elazeim reported that 3 patients (15\%) had pain and erythema at the site of injection, 1 had swelling (5\%), and 
2 had transient post-hypopigmentation (10\%). Sharquie's study had 1 patient (3.3\%) who complained of pain necessitating the intake of non-steroidal anti-inflammatory drug. Amirnia 2015, on the other hand, had no reported adverse events for both intralesional PPD and placebo group but had significant adverse events in the cryotherapy group: pain in 20 patients $(60.6 \%)$, blisters in 11 (33.3\%), erythema in $4(12.1 \%)$, swelling in $2(6.1 \%)$ and hyperpigmented scar in $6(18.2 \%)$. Shaheen 2015 reported that all patients across the different groups complained of pain but in the MMR group, erythema, swelling, and vasovagal attack was seen in 1 patient each (10\% each), while 1 patient in the placebo group also had vasovagal attack.

\section{DISCUSSION}

\section{Summary of main results}

Four controlled clinical trials with a total of 202 patients involved were included in this review. All studies compared the use of intralesional PPD with intralesional saline (placebo) for treating warts but with the addition of intralesional MMR for Shaheen's study and cryotherapy for Amirnia's study. Intralesional PPD is theorized to work by inducing a delayed type hypersensitivity reaction inducing the release of cytokines which will help clear the virus from the system. Studies have been done to prove its efficacy and safety on the treatment of warts since it poses as a good alternative treatment option due to its relatively cheaper cost.

On assessment of the studies' risk for the different kinds of bias using the criteria by Cochrane Collaboration, there was high risk for bias. Most of the studies were unclear of the randomization process and allocation concealment, and did not do appropriate blinding of patients and of assessors compromising the quality or validity of the evidence. There were also high drop-out rates in two of the studies. Because of this, the results must be assessed and applied with wariness and caution.

As for treatment effect, intralesional PPD was significantly more effective when it comes to complete resolution rates of both the target wart and distant wart compared to placebo. However, there was high degree of heterogeneity across the included studies. Factors that might have contributed to this high heterogeneity include clinical diversity, different dosing and intervals of treatment and methodological diversity, absence of blinding in other studies, and issues of allocation concealment. In terms of recurrence rates, there was no difference with placebo.

Most common adverse event reported across the studies was pain. Others included swelling, erythema, and post-inflammatory pigmentation. No major adverse events related with the use of intralesional PPD were reported.

Overall completeness and applicability of evidence

All of the studies were able to address the outcome measures set by this review. Despite the high heterogeneity of the trials included, the treatment effect adjusted accordingly showed that intralesional PPD is an effective option in treating cutaneous warts. However, to make the claims stronger, more studies with better quality or lesser risk for bias are needed. Furthermore, to support the use of intralesional PPD as not just alternative to the treatment of cutaneous warts, more clinical trials are needed, especially those that compare PPD with not only placebo but also with the more conventional therapeutic options.

The population in the studies included children where verruca vulgaris is the most common. Also, males and females were well-represented. These studies were also made in developing countries where BCG vaccination is deemed obligatory, which is one of the inclusion criteria of some of the studies so that the patients will most likely test positive in the intradermal PPD test. As for the intervention, there is still no standard dosing and dosing frequency. For the outcomes, patient satisfaction in terms of efficacy, convenience, and cost was also lacking in the studies available.

Based on the results of this review, intralesional PPD can be used as an alternative treatment option for cutaneous warts. This can be used especially if the conventional therapeutic modalities have already failed since in two of the four studies, they included patients who had multiple recalcitrant warts.

\section{Quality of the evidence}

All studies were of high risk for bias. However, as for the research outcomes, inconsistency, indirectness, and imprecision were evaluated to get the quality of evidence using the GradePRO GDT Software handbook as a guide. ${ }^{9}$

\section{Inconsistency}

Inconsistency is defined as the heterogeneity of the studies that is not explainable. ${ }^{9}$ All the outcomes were assessed to be "not serious" for this category. Although the point estimates of the studies were consistent when it comes to whether intralesional PPD is effective or not, the reason for the high value of heterogeneity must still be explored. One possible reason is that some of the studies recruited specifically patients with recalcitrant warts only. Thus, we can anticipate that they will have smaller treatment effects (Sharquie 2016 and Abd-Elazeim 2014) than the other two studies contributing to the varied confidence intervals and minimizing the overlaps between them.

\section{Indirectness}

Directness refers to a study directly comparing the intervention that the review is interested in, applying this intervention to the population the review is interested in and measuring the outcomes that are deemed significant to the patients. ${ }^{9}$ In this review, all outcomes in the study were assessed to be direct. The population and intervention for 
all trials were all fitting with what this meta-analysis would like to study. As for the outcomes, the studies measured the level of response/clearance of the warts and their recurrence which are also directly observed by patients themselves, making the results of the study applicable.

\section{Imprecision}

For systematic reviews, the quality of evidence when it comes to imprecision can be downgraded for each outcome if the optimal information size criterion is not met unless the sample size is very large or if the OIS criterion is met but the $95 \%$ confidence interval overlaps the null value and fails to exclude important benefit or important harm. ${ }^{9}$

For the first outcome, non-resolution of target wart, the OIS is 9 per treatment group which was adequately met by the study. Also the $95 \%$ CI did not include the RR of 1 making this outcome precise. As for the second outcome, non-resolution of distant warts, the computed OIS is at 15 patients per treatment group which was also adequately met. Again, the 95\% CI excluded the null value. Thus, this outcome was also assessed to be "not serious" for imprecision. For the third outcome, however, the computed OIS is at 1782099 which was not met by this meta-analysis. Sample sizes were only at 85 and 84 for the intralesional PPD group and the placebo group, respectively. Hence, this outcome was evaluated to be "serious" for imprecision.

\section{CONCLUSIONS}

\section{Implications for Practice}

Considering all the evidence presented regarding the efficacy and safety and the quality of this evidence, intralesional PPD can be a good alternative for the treatment of warts especially the recalcitrant ones. Benefits with the use of this treatment modality include its low cost (Php 95 per vial - at most 1 vial for 6 sessions) and the fact that it only needs to be injected in one target wart decreasing the incidence of side effects on all other warts which is seen in other treatment options like cryotherapy. However, patients should not have an immunocompromised condition, and should be willing to follow up multiple times for the treatment to work, which adds to the cost of treatment. Also, patients should have baseline positive reactivity to the intradermal PPD test which is highly likely when patients have already received the BCG vaccination which is obligatory as part of the Expanded Program on Immunization in the Philippines. The paucity of studies, though, that compare this treatment to other more widely used forms of treatment for cutaneous warts makes it a challenge to consider intralesional PPD as one of the firstline treatment options.

\section{Implications for Research}

Since all of the studies that were included in this meta-analysis were assessed to have high risk for bias, trials with better quality and designs are needed. Also, studies to determine the optimal dosing and frequency of treatment are necessary to standardize the intervention across studies, thereby decreasing the potential for other confounding variables. Studies with longer follow-up rates can also be done to further assess the chance of recurrence with the use intralesional PPD and to associate these events with clinical parameters. Lastly, as mentioned earlier, non-inferiority studies comparing intralesional PPD with the conventional modes of treatment are in order for it to be considered as first line of treatment when dealing with cutaneous warts.

\section{Statement of Authorship}

All authors approved the final version submitted.

\section{Author Disclosure}

All authors declared no conflicts of interest.

\section{Funding Source}

This paper was funded by the authors. No external funding agency.

\section{REFERENCES}

1. Wolff K, Goldsmith L, Katz S, Gilchrest B, Paller AS, Leffell D, eds. Fitzpatrick's Dermatology in General Medicine. 8th Edition. New York: McGraw-Hill Companies Inc; 2012. Chapter 196, Human Papilloma Virus Infections; pp. 2421-33.

2. Aldahan AS, Mlacker S, Shah VV, et al. Efficacy of intralesional immunotherapy for the treatment of warts: A review of the literature. Dermatol Ther. 2016;29(3):197-207. doi: 10.1111/dth.12352.

3. Amirnia M, Khodaeiani E, Fouladi DF, Masoudnia S. Intralesional immunotherapy with tuberculin purified protein derivative (PPD) in recalcitrant wart: A randomized, placebo-controlled, double-blind clinical trial including an extra group of candidates for cryotherapy. J Dermatolog Treat. 2016;27(2):173-8.

4. Abd-Elazeim FM, Mohammed GF, Fathy A, Mohamed RW. Evaluation of IL-12 serum level in patients with recalcitrant multiple common warts, treated by intralesional tuberculin antigen. J Dermatolog Treat. 2014;25(3):264-7.

5. Shaheen MA, Salem SA, Fouad DA, El-Fatah AA. Intralesional tuberculin (PPD) versus measles, mumps, rubella (MMR) vaccine in treatment of multiple warts: a comparative clinical and immunological study. Dermatol Ther. 2015;28(4):194-200.

6. Review Manager (RevMan) [Computer program]. Version 5.3. Copenhagen: The Nordic Cochrane Centre, The Cochrane Collaboration, 2014.

7. Cochrane Handbook for Systematic Reviews of Interventions Version 5.1.0 [Internet]. The Cochrane Collaboration; 2011Mar [cited 2017 Jul 10]. Available from http://handbook-5-1.cochrane.org/.

8. Johnson SM, Roberson P, Horn T. Intralesional Injection of Mumps or Candida Skin Test Antigens: A Novel Immunotherapy for Warts. Arch Dermatol. 2001;137(4):451-5.

9. GRADE handbook: Handbook for grading quality of evidence and strength of recommendations [Internet]. The GRADE Working Group; 2013, updated 2015 Dec 04 [cited 2017 Jul 10]; Chapter 5. Available from https://gdt.gradepro.org/app/handbook/ handbook.html. 